\title{
19 (comunitania)
}

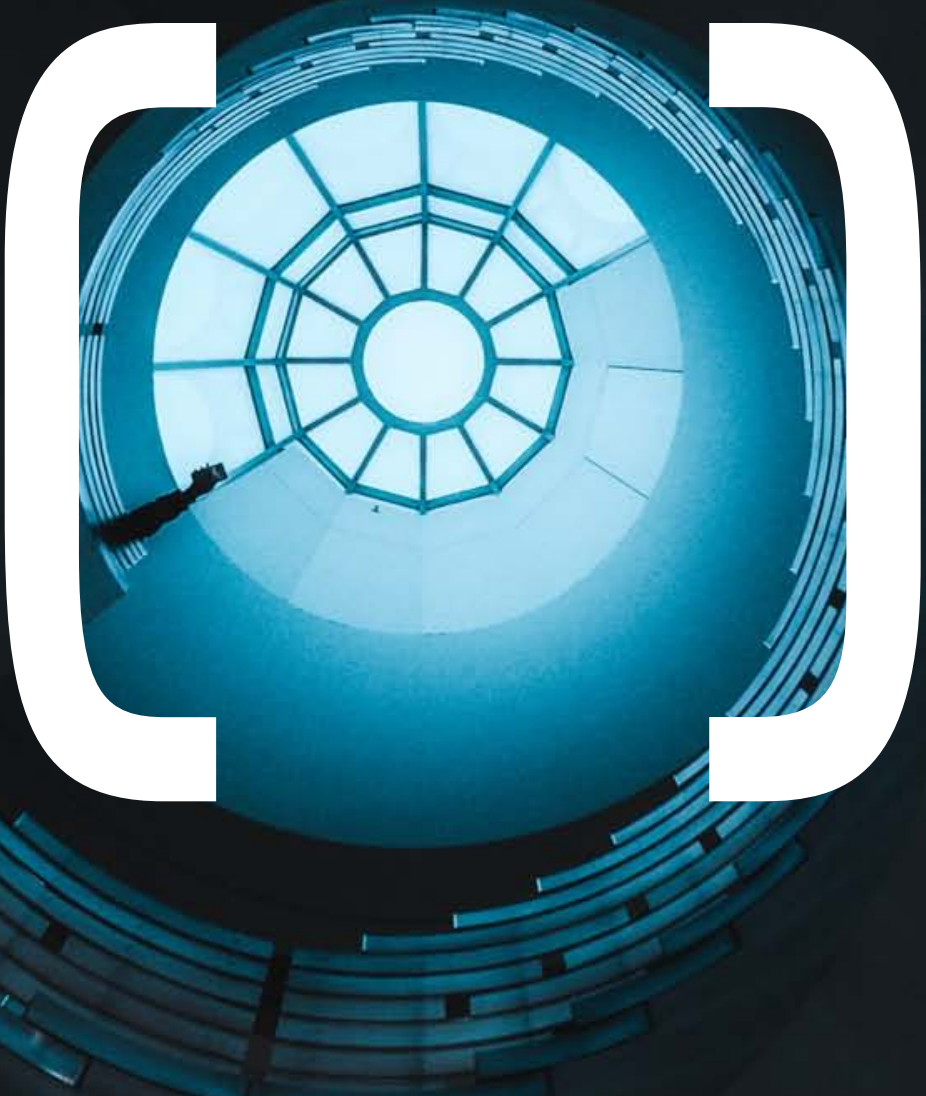

JESÚS ACEVEDO ALEMÁN | KARLA CECILIA CUELLAR FERMÍN | ÁLVARO ELICES ACERO | LEONOR TERESO RAMÍREZ TERESITA DEL NIÑO JESÚS CARRILLO MONTOYA | LUZ MERCEDES VERDUGO ARAUJO | SENADOR PASCUAL LAVILLA MARÍA DEL CARMEN SANTOS FABELO 


\section{RESEÑNA}

\section{Páez Cot, C. 2017. Alas de Papel. Una nueva mirada hacia las personas que sufren trastorno mental / Paper Wings. Another look at persons suffering from mental illness. Barcelona: Espai Literari}

Reseña realizadd por Nuria Hernández Casado

DOI: http://dx.doi.org/10.5944/comunitania.19.7

\section{Reseña:}

Alas de Papel es el primer libro publicado por Cristina Páez Cot, diplomada en trabajo social, master en ecología emocional y posgrado en salud mental e inmigración. Alas de Papel refleja el bagaje de su autora, que cuenta con una trayectoria profesional de más de treinta años, como trabajadora social en el campo de la salud mental en equipos de atención de adultos, directora de la Fundación Privada Salud Mental Catalunya y colaboradora en la Universitat Oberta de Catalunya como docente del Máster deTrabajo Social Sanitario y del Posgrado de Salud Mental Colectiva.

La idea central del libro es hacer un cambio de mirada hacia las personas que sufren trastorno mental, mediante un modelo de atención centrado en el respeto a la persona, a su fragilidad y vulnerabilidad, defendiendo siempre sus derechos fundamentales y creyendo firmemente en sus capacidades y sus posibilidades de cambio y mejora, incluso en casos de pacientes con diagnósticos crónicos de trastorno mental severo. Una atención preventiva, integral, interdisciplinaria, coordinada, en equipo, en red y garantizando la continuidad asistencial. Un modelo que prioriza el vínculo y la relación asistencial con la persona afectada y sus familiares, sin olvidarse de su comunidad.

El libro se inicia con un poema en catalán del hijo pequeño de la autora, que precisamente se titula Con otros ojos, y destila una visión de la vida realista y optimista. Continúa con un magnífico prólogo escrito por José Leal Rubio -psicólogo clínico, psicoanalista, supervisor clínico e institucional-, donde resume la esencia de la obra, destaca el fuerte compromiso ético y profesional de la autora y pone de manifiesto la necesidad de profundizar en prácticas innovadoras por el impacto que pueden conseguir. 
Encontramos una extensa introducción que contiene la tesis del ensayo y nos introduce en las ideas que se irán desarrollando a lo largo de los siete capítulos en que se estructura, transmitiendo la invisibilidad del trabajo social especializado en salud mental y resaltando la potencia de la herramienta del trabajo en el domicilio. El libro se completa con unas reflexiones finales y unos generosos agradecimientos que incluyen a referentes personales, profesionales $y$, sobre todo, a los pacientes $y$ sus familias. Por último, cuenta con una extensa bibliografía y un completo marco legal que permitirán profundizar en los distintos temas planteados.

Todos los capítulos cuentan con contenidos teóricos, análisis, reflexiones y casos prácticos con detalladas y conmovedoras descripciones de personas, contextos, domicilios, familias y emociones. Todos los casos son ejemplos de buenas prácticas de metodología, ritmos, cuidado y respeto profesional. Cada apartado finaliza con unas conclusiones y apunta, con buen criterio, líneas de intervención futuras.

A lo largo de Alas de Papel, la autora va redundando en la idea central del cambio de mirada, al tiempo que analiza y cuestiona políticas, sistemas, organizaciones, prácticas y posicionamientos técnicos, defendiendo que deberían ser horizontales -entre profesionales, entre servicios y con la persona atendida y sus familiares-. Al mismo tiempo, rompe una lanza por los profesionales, su buen hacer y su ilusión por la mejora continua, entendiendo que la presión asistencial y los miedos ante pacientes con tanto riesgo social, conducen muchas veces a intervenciones que no son las más indicadas. Incide en todo lo que desde el trabajo social se puede y debe tratar de gestionar: emociones, información, miedos, fantasías, confianza, esperanza....Por último, deja muy claro que la Administración (sanitaria, social, educativa, de justicia...) debe dotar a nuestra sociedad de leyes, organizaciones y financiación que garanticen los derechos de pacientes y familiares.

El tono del ensayo es de empatía, porque reconoce la complejidad de las actuaciones y las limitaciones de recursos existentes, pero también encontraremos crítica y autocrítica sin paliativos. Páez pide valentía para afrontar todos los temas que se deben abordar y para potenciar la autonomía de la persona, siempre en base a las necesidades y deseos de cambio que ésta exprese, asumiendo los riesgos que sean necesarios. Describe las dificultades inherentes a profesionales del trabajo social, de la enfermería, de la medicina familiar o especializada y de la psicología o la psiquiatría, donde se debe trabajar teniendo en cuenta que "cada profesional es especialista en una parte del todo y todas las partes son importantes" (p. 53 ). Observaremos algunas referencias etimológicas a las que a Páez le gusta recurrir porque, para ella, las palabras encierran un gran significado y sentido.

El primer capítulo lleva por título Trabajo social en el hogar; espacio de reflexión y cambio. El hogar es donde, a menudo, la persona con trastorno mental se aísla y donde el trabajador social debe dirigirse con una actitud proactiva y generadora de cambios. Es un espacio básico de creación de vínculo y confianza, en cualquier fase de la intervención. 
El segundo capítulo trata del impacto del diagnóstico y el acompañamiento emocional entre los 16 y los 24 años, una etapa de vital importancia para la persona. Analiza el vacío entre el alta de los servicios de salud mental infantojuvenil a los 18 años y los 24 ó 25 años en que las mismas personas aparecen en los servicios de adultos, con el deterioro que se puede haber producido en ellas y sus familias durante ese tiempo. Desgrana emociones y reacciones tales como rechazo, dolor, soledad, desorientación y espanto, que se producen en pacientes y familias en el duelo que supone un diagnóstico que condena y frustra la mayoría de ilusiones y proyectos vitales.

El siguiente apartado está dedicado a la madres, eternas cuidadoras. Páez se pregunta: ¿de quién, de qué, hasta cuándo y por qué?. Nos describe la dura invisibilidad de las madres cuidadoras que "no se quejan, ni claudican, ni delegan" y de los sentimientos de culpa que arrastran toda su vida. Desde una perspectiva histórica y feminista, analiza el rol de cuidadora asignado al género femenino, cómo afecta a la salud de las mujeres y la necesaria corresponsabilidad social e institucional para mejorar los cuidados y compensar esta dedicación.

El libro tiene un capítulo dedicado íntegramente a hablar de soledad, afectividad $y$ sexualidad, temas que se deben hacer emerger ya que afectan directamente a derechos fundamentales como la dignidad, la igualdad, la libertad y la intimidad de las personas. Se debe ofrecer diálogo y escucha, con sensibilidad y elegancia, y ayudar a las familias a ver los beneficios y no las amenazas de promover el desarrollo de estos ámbitos vitales.

Otro tema que merece una sección en Alas de Papel es el de la protección legal. Bajo el título ¿Acompañar, proteger o incapacitar?, analiza cómo los profesionales muchas veces actúan por miedo, angustia y preocupación por el futuro de los pacientes y su entorno, así como por desconocimiento hacia las diferentes figuras jurídicas. Considera que se ha abusado mucho de los procesos de incapacitación, ineficaces en muchos casos, y que suponen un gran coste emocional y económico.

En el siguiente capítulo se reflexiona sobre el futuro de las personas con trastorno mental severo que viven con sus padres mayores, situación de gran prevalencia dada las escasez de recursos públicos destinados a promover su independencia. Es preciso ayudar a pensar cómo quieren que viva su familiar cuando ya no le puedan atender y hacer partícipe a la persona afectada, potenciar su autonomía, superar dinámicas de sobreprotección así como miedos o fantasías sobre lo que puede llegar a ocurrir, y prever la claudicación ante unos procesos crónicos muy duros.

Por último, Alas de papel se pregunta ¿Por qué se nos mueren tan pronto? y analiza las dificultades de articulación entre la red de salud mental y atención primaria de salud. Aporta datos estadísticos sobre enfermedad y baja esperanza de vida de estas personas, con unos servicios colapsados y diseñados sin tener en cuenta las características de esta población al tiempo que recalca la importancia de generar 
una mayor confianza y mejores circuitos de coordinación y derivación entre los servicios sanitarios.

Por su rigurosidad y especificidad, se puede considerar una obra de referencia en el trabajo social que será muy útil a profesionales de los ámbitos social o sanitario, sea cual sea su nivel de responsabilidad. Muy adecuada también a nivel académico $y$, por su lenguaje asequible y próximo, su lectura puede ayudar también a las personas con trastorno mental y sus familiares.

Alas de Papel se publicó en catalán y posteriormente se tradujo al castellano. Está disponible en papel y digital, con una segunda edición revisada y publicada en 2019. En breve aparecerá la primera edición en inglés. La obra ha sido presentada en diferentes ciudades de España y se encuentra en bibliotecas de prestigiosas universidades y centros culturales de interés, como la biblioteca del Parlamento Europeo, donde se presentó el pasado mes de abril.

Finalizaremos esta reseña con una última cita de la autora que resume su filosofía, ética profesional y respeto absoluto a la persona: "Seamos osados y propongamos intervenciones con la persona, pero, primero, dejémosle a ella su protagonismo. Dejémosle ser protagonista de su vida" (p.231). 


\section{comunitania}

REVISTA INTERNACIONAL DE TRABAJO SOCIAL Y CIENCIAS SOCIALES

INTERNATIONAL. JOURNAL OF SOCIAL WORK AND SOCIAL SCIENCES

\section{ARTICULOS/ARTICLES}

El lado oculto del bullying: los espectadores. Retos del trabajo social / The hidden side of bullying: the spectators.

Challenges of social work

Jesús Acevedo Alemán y Karla Cecilia Cuellar Fermín

Págs 9-27

El componente de la activación laboral en la Renta Garantizada de Ciudadanía de Castilla y León: una perspectiva profesional / The component of the work activation in the Guaranteed Income of Citizenship of Castile and Leon:

a profesional perspective

Álvaro Elices Acero

Págs 29-44

Rompiendo cotidianidades y tejiendo redes comunitarias para desarrollar empoderamiento dialógico-colectivo /

Breaking everyday and weaving community networks to develop dialogical-collective empowerment

Leonor Tereso Ramírez, Teresita del Niño Jesús Carrillo Montoya y Luz Mercedes Verdugo Araujo

Págs 45-55

Familias multiproblemáticas y el programa de apoyo a familias en Soria / Multi problem families and the family

support program in Soria

Senador Pascual Lavilla

Págs 57-78

La educación especial en Ciudad Juárez: reflexión y acción / Special education in Ciudad Juárez: reflection and action María del Carmen Santos Fabelo.....

Págs 79-93

\section{RESENIAS/REVIEWS}

Castillo de Mesa, J. 2019. El Trabajo Social en la era digital / Social Work in the Digital Era (por Paula Méndez Domínguez)

Págs 93-97

Páez Cot, C. 2017. Alas de Papel. Una nueva mirada hacia las personas que sufren trastorno mental / Paper Wings. Another look at persons suffering from mental illness

(por Nuria Hernández Casado) 